\title{
Laboratory Behavior of a Plasma Plume Injected into the Magnetized Plasma Flow
}

\author{
Shigeyuki Minami ${ }^{1}$, Peter J. Baum ${ }^{2}$, George Kamin ${ }^{3}$, Yoshio Takeya ${ }^{4}$, \\ and R. Stephen WHITE \\ ${ }^{1}$ Department of Electrical Engineering, Osaka City University, Sumiyoshi, Osaka 558, Japan \\ ${ }^{2}$ General Research Corporation, Santa Barbara, CA 93102, U.S.A. \\ ${ }^{3}$ Sandia Laboratory, INC., Albuquerque, NM 87116, U.S.A. \\ ${ }^{4}$ Department of Electrical Engineering, Chubu University, Kasugai, Aichi 487, Japan \\ ${ }^{5}$ Institute of Geophysics and Planetary Physics, University of California, Riverside, CA 92521, U.S.A.
}

(Received February 6, 1988; Accepted July 11, 1988)

\begin{abstract}
Laboratory plasma plumes injected into magnetized plasma flows simulate the formation of cometary magnetospheres. Likewise, in our laboratories plasma plumes are injected into the tail of the simulated earth's magnetosphere produced by an interaction between the simulated solar wind and a magnetic dipole. The behavior of laboratory artificial plasma plumes injected into the magnetized plasma flow is discussed in conjunction with the AMPTE artificial comet experiments and other active chemical release experiments in space.
\end{abstract}

1. Simulation of the Earth's Magnetosphere and Plasma Plume Injections into the Tail

We have investigated the earth's magnetosphere in the laboratory using the interaction of a high-speed plasma flow with a magnetic dipole field called a "terrella experiment" by BIRKELAND (1908). He carried out the first solar terrestrial laboratory simulation experiments of the solar corona, aurora and comets. Early experiments by BosTICK et al. (1963), CLADIS et al. (1964), KAWASHIMA (1964) and OSBORNE et al. (1964) focused attention on the formation of a collisionless shock front and of a magnetospheric cavity. These experiments were possible after the improvement of the high-speed, high density plasma gun to simulate the solar wind. A successful injection of plasma into the nightside through the dayside cusp was one of the achievements of such laboratory simulation experiments (KAWASHIMA and MORI, 1966; PODGORNY, 1976) that are related to the micro non-equilibrium at the shock boundary (BAUM and BRATENAHL, 1983b).

The magnetic field lines in the simulated magnetosphere were mapped using a magnetic probe, and a uniform external field corresponding to the interplanetary magnetic field (IMF) is applied. These experiments proved the existences of open and closed magnetospheres (PoDGORNY, 1976; DUBININ et al., 1977, 1978; BAUM and BRATENAHL, 1982a, 1983a; BAUM, 1984). Although the satellite measurements revealed some parts of the magnetotail structure rather clearly, such laboratory 
terrella experiments still play powerful roles for understanding the global behavior of the magnetosphere when the particular parameters are controlled. The goal of our magnetospheric study is to recognize the role of each of the geophysical parameters for magnetosphere formation.

In addition to the laboratory study of the magnetosphere, another active experiment was conducted by MINAMI et al. (1977) and MINAMI and TAKEYA (1985) using a small plasma injecter. A small discharge electrode set in the nightside

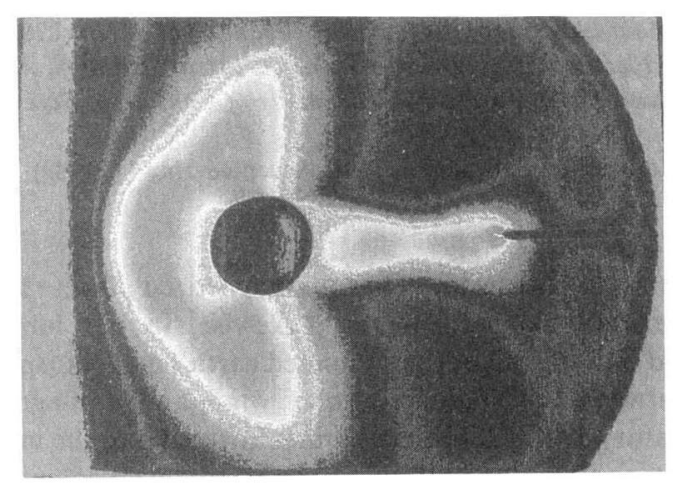

(a) $B_{Z}<0$

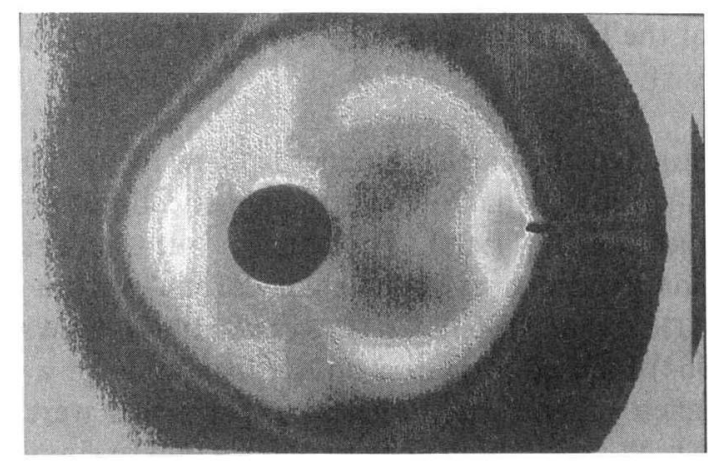

(b) $B_{Z}>0$

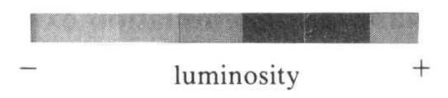

Fig. 1. A set of digitized photographs show plasma density distributions of simulated magnetospheres with oppositely directed interplanetary magnetic fields, $B_{Z}$. (a) The southward IMF corresponds to -5 gamma, i.e., $100 \mathrm{G}$ in the laboratory. (b) The northward IMF corresponds to +5 gamma. All other solar wind parameters are the same (MINAMI and TAKEYA, 1985). The plasma confined inside the magnetosphere comes from the solar wind. So the images are evidence of direct IMF control of the solar wind kinetic energy transfer into the magnetosphere. A small plasma emitter located on the right-hand side was used to trace the magnetic field lines. 
magnetosphere region of the plasma chamber makes possible the tracing of the configuration of the magnetic field lines. The injected plasma plume enables the field lines to be mapped by illumination. Figure 1 shows the confinement of the plasma inside the magnetosphere with (a) a southward IMF, and (b) a northward IMF using a digitized color coding of the black and white time exposure photographs of the simulated magnetosphere (MINAMI and AKASOFU, 1986; MINAMI, 1987a). The intense plasma comes from the left. The result indicates that the plasma energy could enter the magnetosphere effectively when the IMF is directed southward, -5 gamma (scaled), rather than northward, +5 gamma (scaled). The typical solar wind plasma stream from the plasma gun has a density of $10^{14} \mathrm{~cm}^{-3}$ and a velocity of $5 \times 10^{6} \mathrm{~cm} / \mathrm{s}$. The detailed experimental parameters and the reasonable degree of similitude to the magnetosphere were discussed (MINAMI and TAKEYA, 1985, 1986).

The experimental setup of the apparatus is illustrated in Fig. 2. The model earth is a sphere of $35 \mathrm{~mm}$ diameter to which a pulsed magnetic field of about $8 \mathrm{KG}$ is applied. Its duration (about $0.5 \mathrm{~ms}$ ) is much longer than that of the pulsed solar wind lifetime $(70 \mu \mathrm{s})$. Also this plasma lifetime is long enough to simulate magnetospheric phenomena (FUKUSHIMA and KAWASHIMA, 1964). Thus the experimental magnetosphere is quasi-stationary. In Fig. 1, the plasma emitter

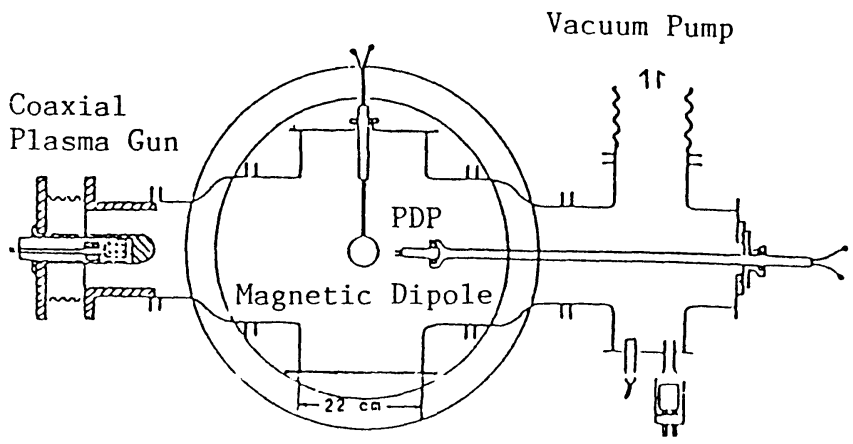

(a) Top View

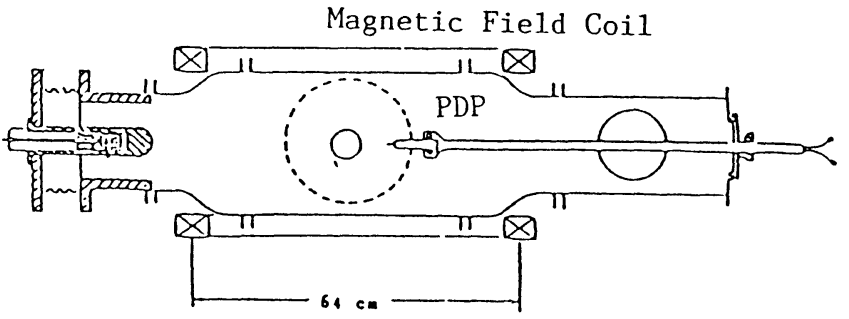

(b) Side View

Fig. 2. Illustration of the setup of the experimental apparatus at Osaka City University: (a) top view, (b) side view. 
illuminates a part of the tail of the nightside magnetosphere on each photograph showing the injected plasma plume. The typical density, $N$, and duration of the injected plasma are $10^{12} \mathrm{~cm}^{-3}$ and $10 \mu \mathrm{s}$.

Figures 1(a) and (b) present evidence for (a) open and (b) closed structures of magnetic field lines in the simulated magnetosphere. The magnetospheric simulation experiment by MINAMI and TAKEYA (1985) reveals not only the quantitative energetic coupling of the magnetosphere and the solar wind for particle energy transport (MINAMI and AKASOFU, 1988) but also the usefulness of such a plasma plume injection method as a tracer of magnetic field lines. The duration of the plasma plume is short enough to detect the dynamical behavior of magnetic field lines as if it is illuminated by a flash bulb. Figure 3 also shows the configuration of magnetic field lines which cross the tail region at a geocentric distance of $18 \mathrm{RE}$. The distance is scaled by the ratio of the stand off distance of the actual (10 RE) and simulated magnetospheres $(3 \mathrm{~cm})$. It could be observed that injected plasma
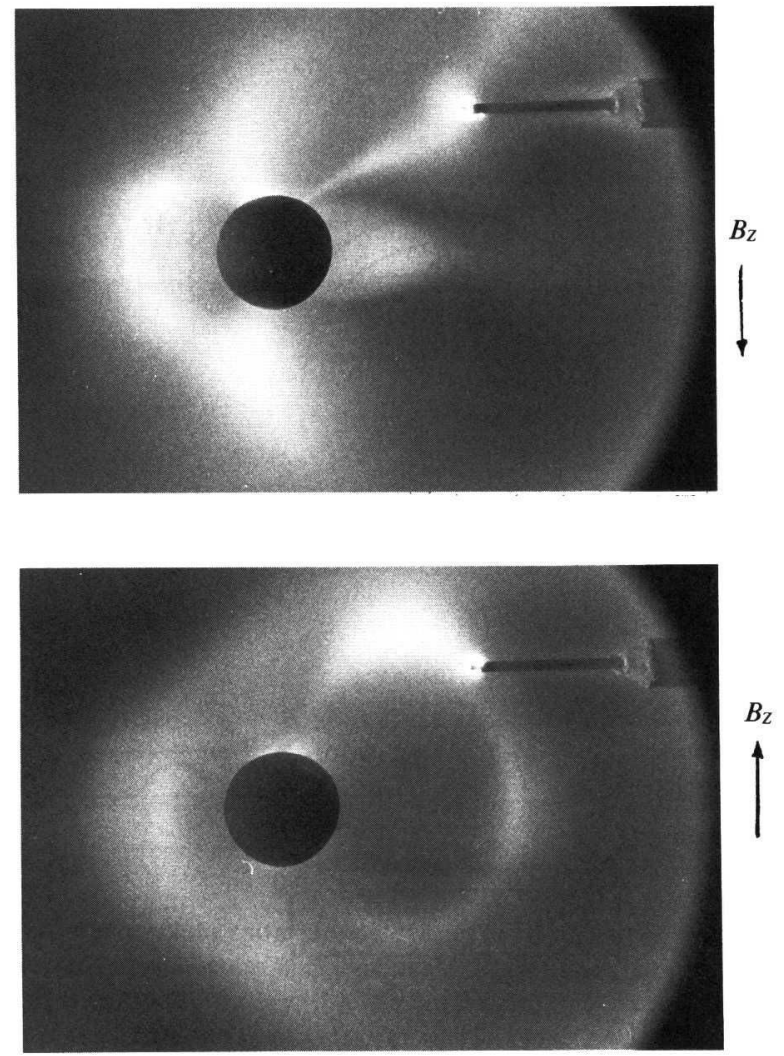

Fig. 3. Experimental results of the magnetic field line tracing by a plasma emitter using two opposite directions of $B_{Z}$ (MINAMI and TAKEYA, 1985). 


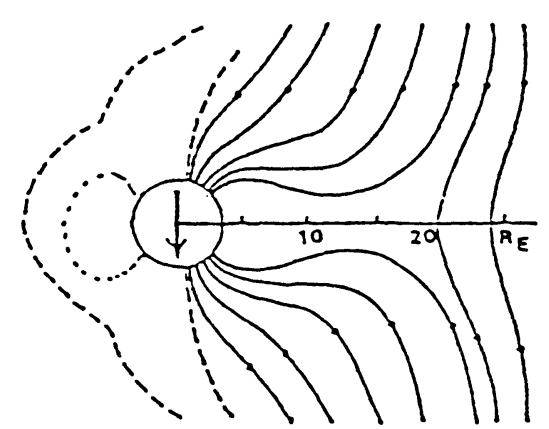

[4]

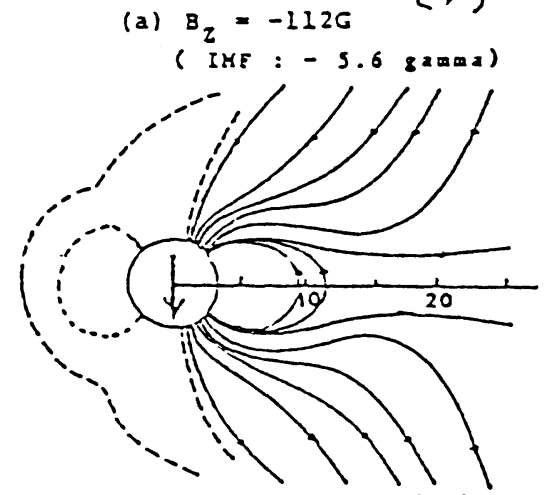

(b) $B_{2}=-560 \quad[t]$
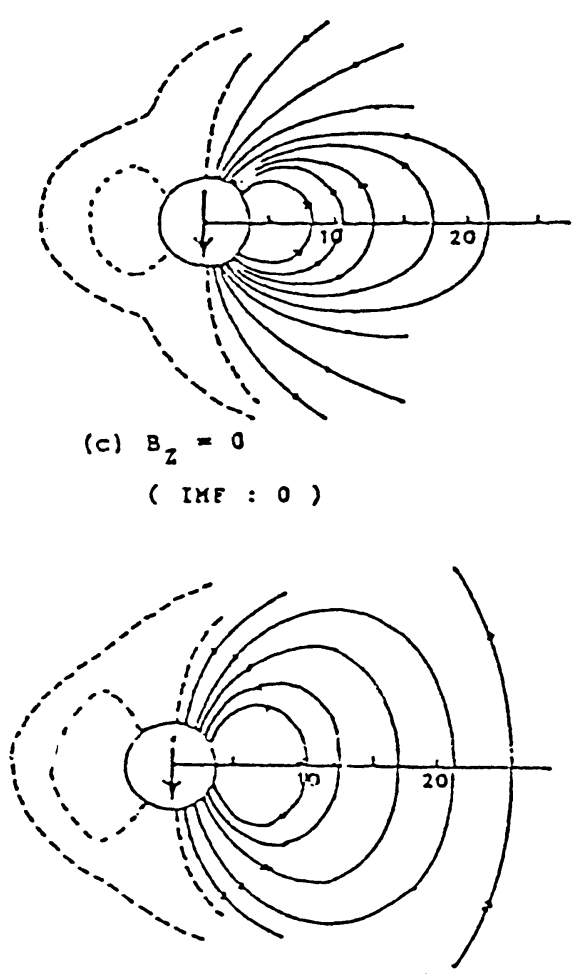

(d) ${ }_{3}=500 \quad[t]$ (IMF : $-2.3 \mathrm{gamm}$ )

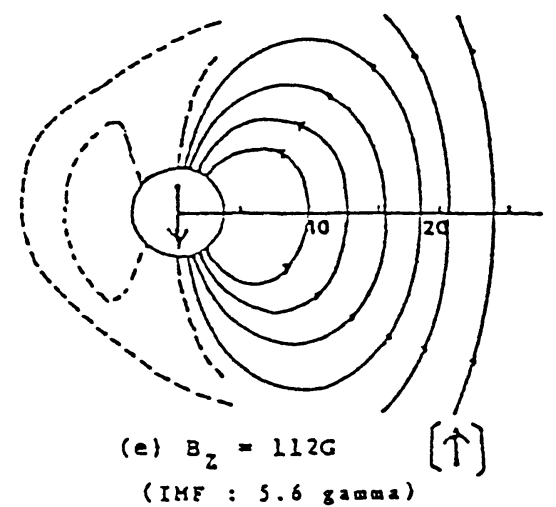

Fig. 4. Summarized results of observed magnetic field lines by the plasma plume injection. 
produced by the local discharge of the ambient neutral gas moves along the magnetic field lines which pass through the position of the plasma emitter and impacts the model earth. The behavior of the plasma plume verifies the field line tracing by the chemical release experiment in space (HAERENDEL, 1973). The results of traced magnetic field lines in a simulated magnetosphere support the theory of fundamental magnetic field reconnection which occurs when the plasma flow passes through the magnetosphere. DUNGEY (1962) showed that the external magnetic field is connected with the magnetosphere. A summary of the results of observed magnetic field lines traced by artificial plasma injection at different positions on the sun-earth meridional plane is shown in Fig. 4.

Instead of the discharge of ambient neutral gas as an emitted plasma plume, vaporized $\mathrm{C}, \mathrm{Ba}$ and $\mathrm{Sr}$ have been used in a large space chamber, UCR-T1, located at the University of California, Riverside. When the carbon plasma is injected into the nightside of the simulated magnetosphere, with southward IMF, an auroral oval was traced by the illumination as shown in Fig. 5.

To demonstrate the characteristics of the diffusion of the emitted plasma more clearly, an optical interference filter with a very narrow band width was used. The set of pictures in Fig. 6 shows the usefulness (a) without, (b) with, the interference filter, by indicating the fine structure of the emitted plasma. We would also suggest that the plasma injection techniques, such as those used for the simulated magnetosphere, could be used as artificial tracers for magnetospheric structures in space.

The detection of the magnetospheric boundary is also a very interesting item. We set several threads in the simulated magnetosphere as shown in Figs. 7(a) and (b). The plasma parameters used in the deflagration mode plasma gun (CHENG,

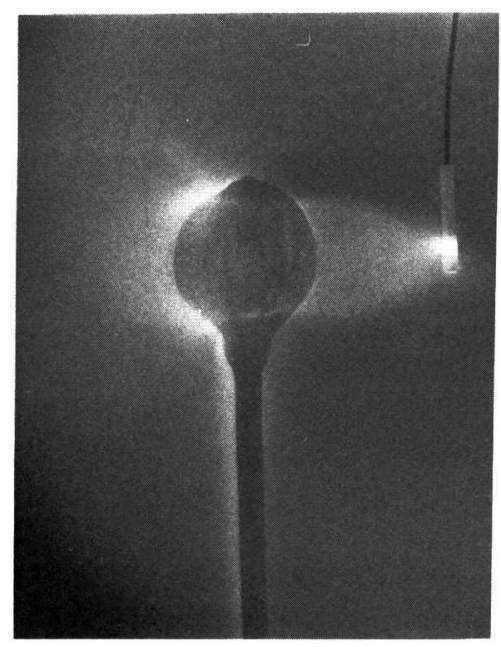

Fig. 5. Carbon plasma injection into a simulated magnetotail using the UCR-T1 $\left(B_{Z}=250 \mathrm{G}\right.$, solar wind plasma density $=10^{12} \mathrm{~cm}^{-3}$ ). 
1971) $(520 \mu \mathrm{F}, 20 \mathrm{kV})$ is nearly the same as that by MINAMI and TAKEYA (1985), (BAUM and BRATENAHL, 1982a). When a thread is set in the nightside region parallel to the $y$-direction (Fig. 7(a)) two illumination points reveal the possible position of the magnetospheric boundary. By setting several threads parallel to the $z$-direction at the front side of the magnetosphere, the magnetospheric shock front is illuminated clearly (Fig. 7(b)). We imagine how spectacular the illumination would be if very long threads could be passed through the magnetosphere. We would say that the visible structure is often more informative than measurements by electrical or magnetic instrument.

\section{Plasma Plume Injection into a Magnetized Plasma Flow}

A magnetized plasma flow such as the solar wind interacts with different obstacles forming interesting structures. Among them, only the cometary magnetosphere is visible to us. One of our purposes in studying the interaction between the magnetized plasma flow and the cometary plasma plume is to understand the acceleration of cometary plasma by the solar wind plasma flow. The other is to learn about the behavior of the plasma plume and the three dimensional structure of the magnetic field related to the artificial plasma injection experiment in space (VALENZUEla et al., 1986). The simulation experiment was carried out with the UCR-T1 space simulation chamber (dia. $1.3 \mathrm{~m}$, length $11 \mathrm{~m}$ ).

Recent observations of comets have furnished a lot of information about the structure of cometary magnetospheres. Especially the apparition of Comet Halley provided a special opportunity for testing theories of the interaction of a comet with the solar wind. Even so more study is necessary, especially as the acceleration and the relationships between the local irregularities and the spatial distributions of the solar wind have not yet been well confirmed. Recently an acceleration mechanism for cometary plasmas using the $J \times B$ force was introduced by MINAMI and WHITE (1986). The calculated results of the distance of the kink from the nucleus, $x$, vs. time, $t$, or velocity of the plasma, $v$, confirmed fairly well the actual cometary observations (TOMITA et al., 1987).

We have also tried to represent an artificial comet in the laboratory using a high-speed plasma flow and injected plasma plume. As we are interested in the formation of cometary plasma tails influenced by the solar wind parameters, such a direct interaction has been studied. The UCR-T1 has an externally applied magnetic field coil simulating the IMF perpendicular to the solar wind plasma flow as shown in Fig. 8. The simulated solar wind (BAUM and BRATENAHL, 1982a) interacts with the plasma released by the plasma emitter (Fig. 9) located about $3 \mathrm{~m}$ downstream from the gun. The plasma plume consists of a tiny plasma gun $(2.3 \mathrm{~mm}$ in dia.) that produces an initial plasma flow from a vaporized teflon insulator. The $\mathrm{Ba}, \mathrm{Sr}$, or $\mathrm{C}$ plasma is produced by an initial ionization of teflon plasma and diffuses through the hole as shown in Fig. 9. Its density $\left(10^{14} \mathrm{~cm}^{-3}\right.$ at $5 \mathrm{~cm}$ from the hole) is measured by a diamagnetic probe. The emitter is very small compared with the shock distance so that an interaction occurs between the plasma flow and the emitted plasma 

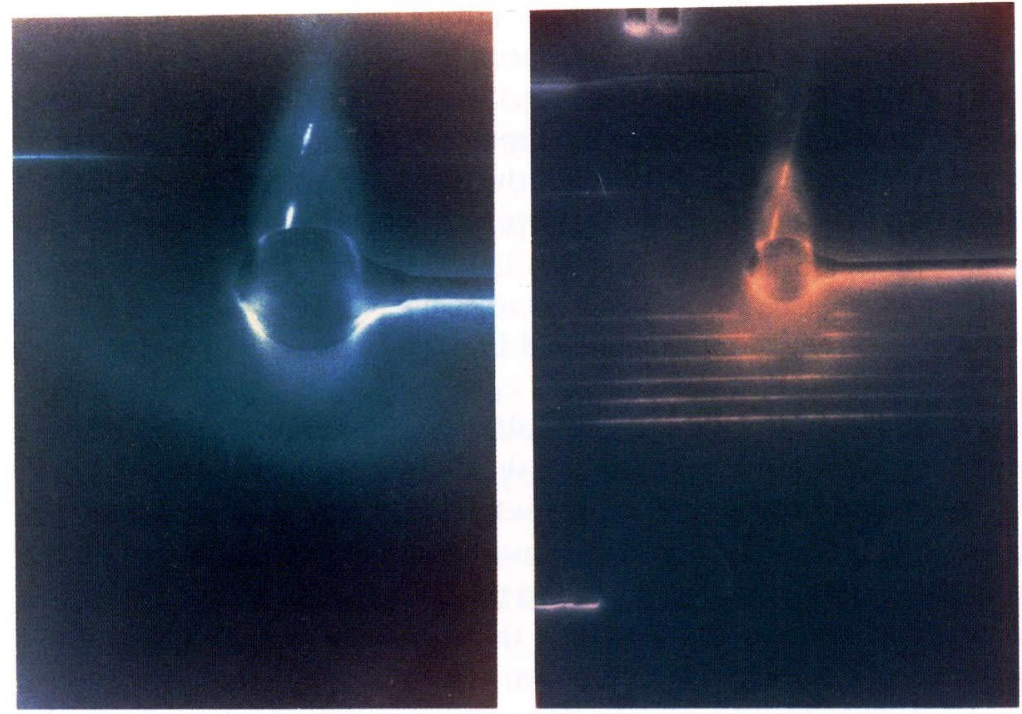

త

$\hat{e}$
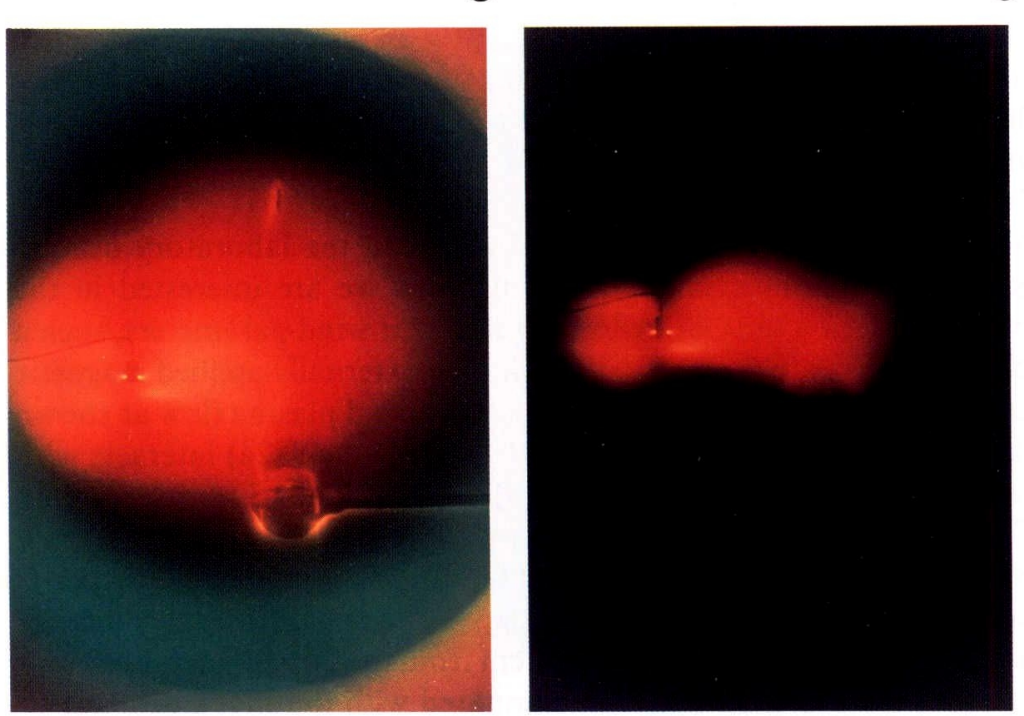

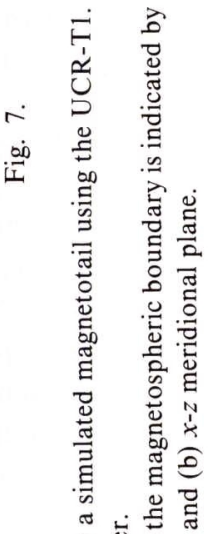

号总号䒕

क \& 3

ह

离

콜 岂

웡

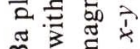

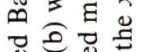

沿

吆

를 $\vec{E}$

政

- 5

总壳 弆

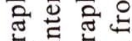

क人요

응 융.

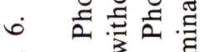

离

这 完 


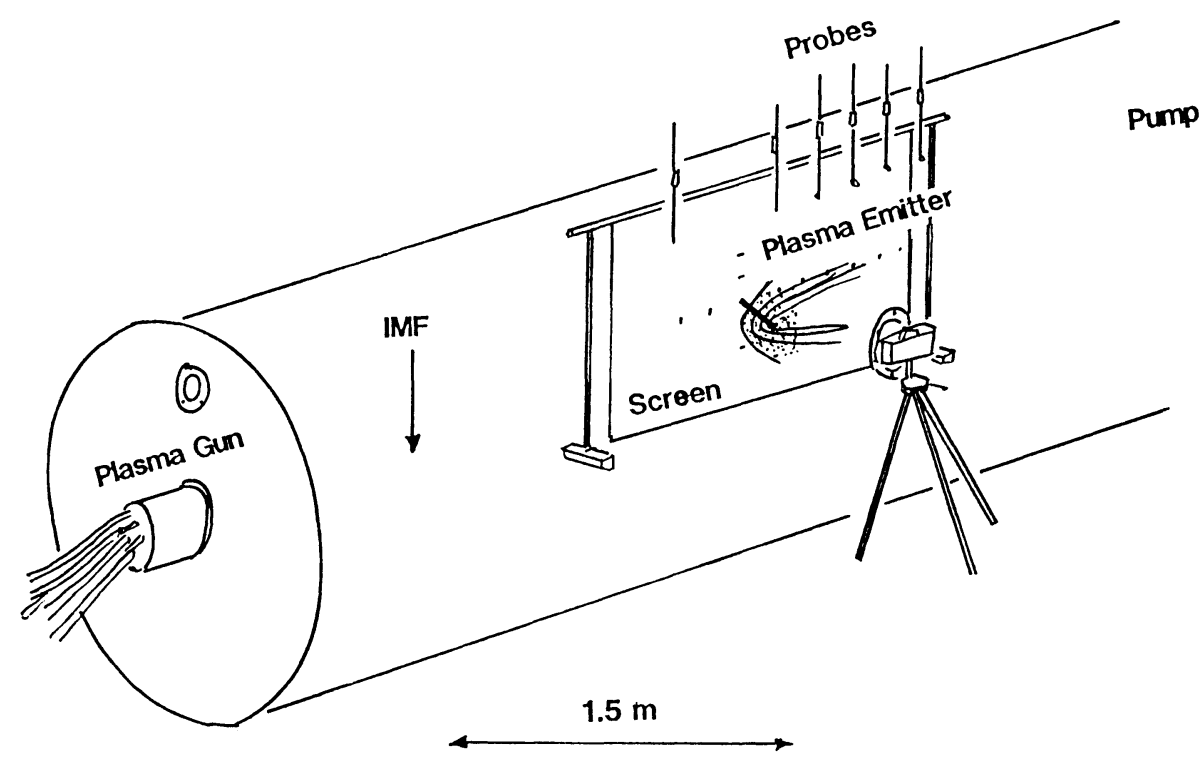

Fig. 8. Experimental setup of the apparatus as part of the UCR-T1. The screen behind the plasma emitter is used to project the spatial scale.

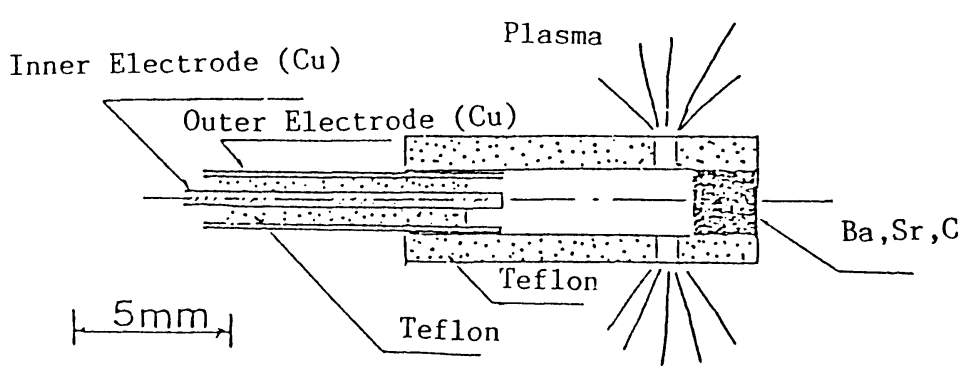

Fig. 9. Configuration of the plasma emitter. The subsonic plasma plume is emitted from a number of holes.

(MINAMI et al., 1986). It is shown that the IMF plays an important role in comet tail formation. For no IMF, Fig. 10(a), the Ba plasma released toward the plasma flow coming from the left-hand side diffuses without any interaction with the plasma flow. The ion-neutral mean free path between the plasma flow and the released plasma from the simulated nucleus is $50 \mathrm{~cm}$, which is long enough for the plasma to diffuse without interaction. For an IMF of $200 \mathrm{G}$, Fig. 10(b), however, the released plasma is trapped and deflected into the cometary tail by the solar wind plasma flow as evidenced by the formation of a shock front.

In Fig. 11, the formation of cometary coma composed of Sr plasma injected 

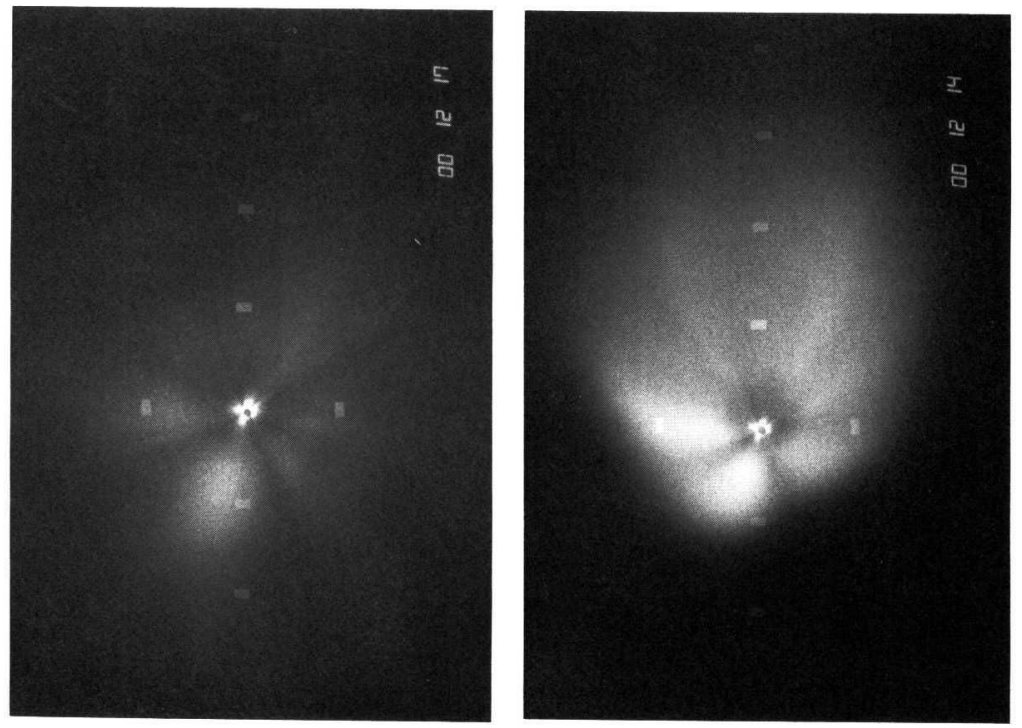

త్ర

อ

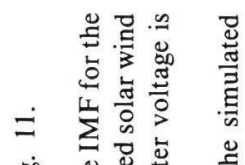

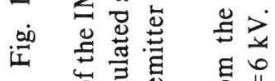

ठ․

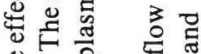

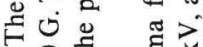

ن 욜 है

छ त ह

등

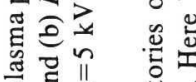

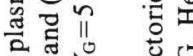

उٓ

光氜

동. ำ

తิ
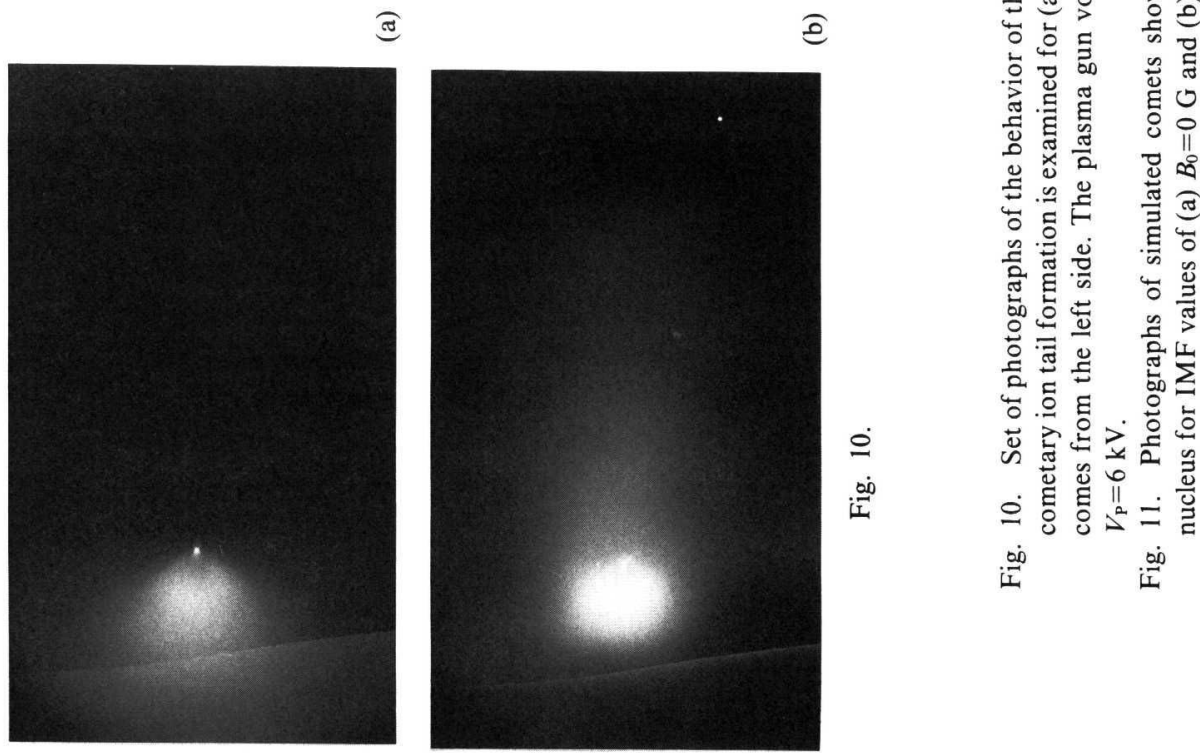

$\infty$ 
from the multi-hole plasma emitter is shown for two different IMF's. The scale shown on the photograph is $7 \mathrm{~cm}$ (division) ${ }^{-1}$ projected onto the sun-comet line. The plasma plume is released through a hole with subsonic perpendicular ram velocity component and IMFs of 0 and $300 \mathrm{G}$. When the IMF is not zero an asymmetrical cometary magnetosphere is observed as shown in Fig. 12(b). The ram pressure of the plasma flow is changed by varying the applied voltage of the plasma gun to study the ionopause formation caused by the interaction between the solar wind and the plasma plume near the nucleus. A set of photographs of simulated cometary structure in Fig. 13 represent the inner coma configuration dependence on $V_{\mathrm{G}}$ (applied gun voltage) at a constant IMF. The data indicate that the solar wind ram pressure controls the stand off distance of the cometary ionopause. The stronger the ram pressure $\left(V_{\mathrm{G}}\right)$, the smaller the stand off distance.

The total plasma content of the injected plasma plume in the inner-coma depends on the applied capacitance bank voltage of the plasma emitter, $V_{\mathrm{P}}$ (MINAMI

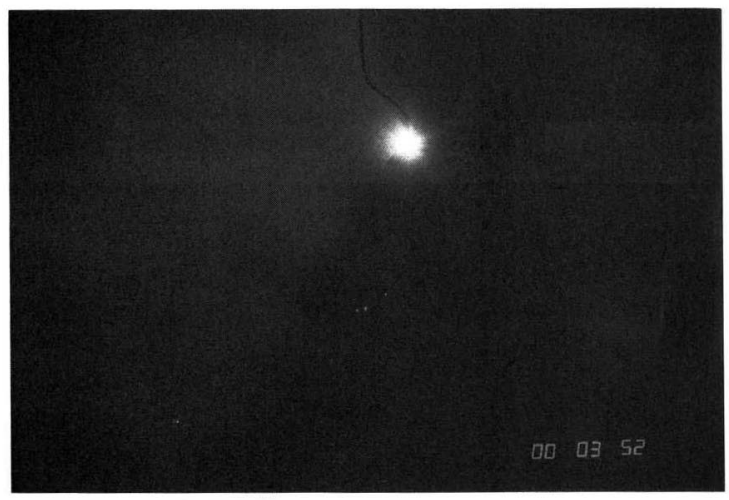

(a)

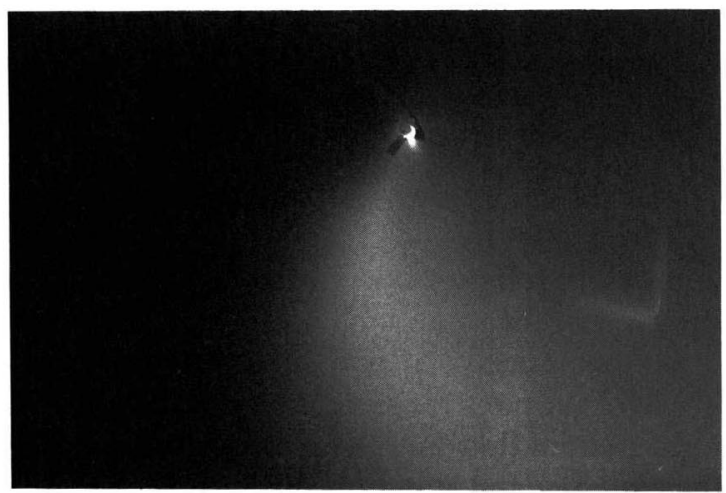

(b)

Fig. 12. Photographs showing evidence for possible asymmetrical structures in a cometary magnetosphere. The plasma plume is injected through a hole perpendicular both to the simulated solar wind plasma flow and the line of sight. (a) $B_{0}=0 \mathrm{G}$, (b) $B_{0}=200 \mathrm{G}$. Here $V_{\mathrm{G}}=5 \mathrm{kV}$. 


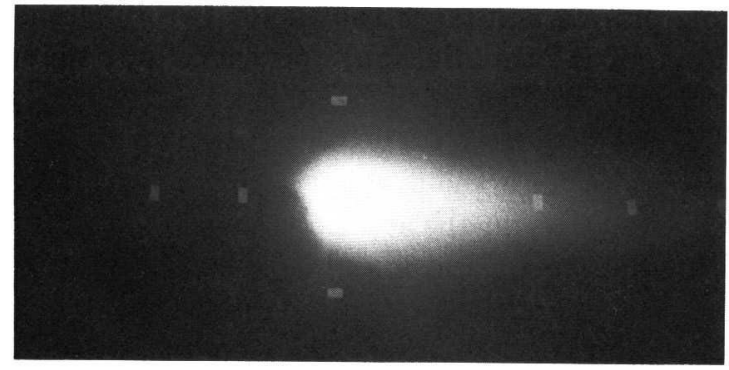

(a)

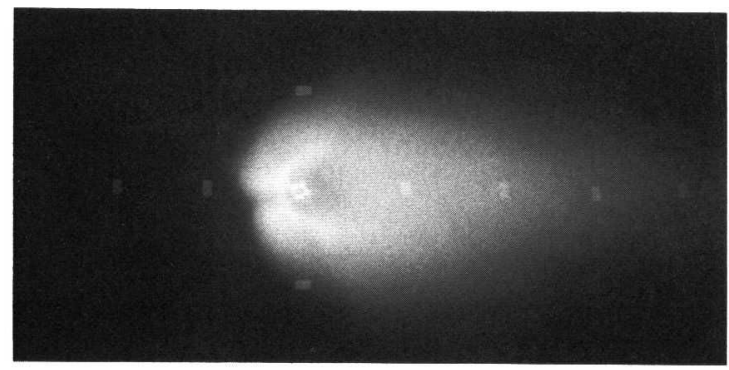

(b)

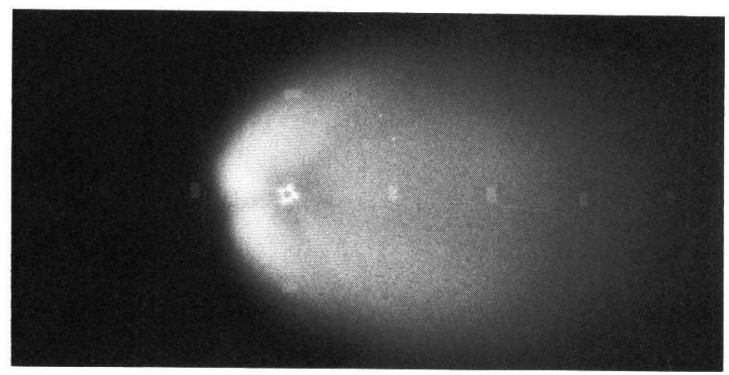

(c)

Fig. 13. Photographs of simulated comets to examine the effect of the solar wind ram pressure by changing the operating voltage of the plasma gun, $V_{\mathrm{G}}$, (a) $7 \mathrm{kV}$, (b) $6 \mathrm{kV}$, (c) $5 \mathrm{kV}$. The IMF is $B_{0}=300 \mathrm{G}$, and the plasma emitter voltage is $V_{\mathrm{P}}=6 \mathrm{kV}$.

et al., 1988), so that the stand-off distance is also controlled by $V_{\mathrm{P}}$ pressure-balanced between the ram pressure of the solar wind and the production rate of the emitted plasma, as is predicted theoretically (IP and AXFORD, 1982). An example of the $V_{\mathrm{P}}$ dependence on the size of the cometary magnetospheric boundary is shown in Fig. 14 using the hydrogen plasma flow and the plasma plume made from carbon. The resultant plot of the experimental stand-off distance versus the operating voltage, $V_{\mathrm{P}}$, is shown in Fig. 15. Note that the stand-off distance (shock front) itself is invisible, even in the case of a visible comet because the luminous neutral coma masks the ionized inner coma in the structure and the shock boundary. 
호

(2)
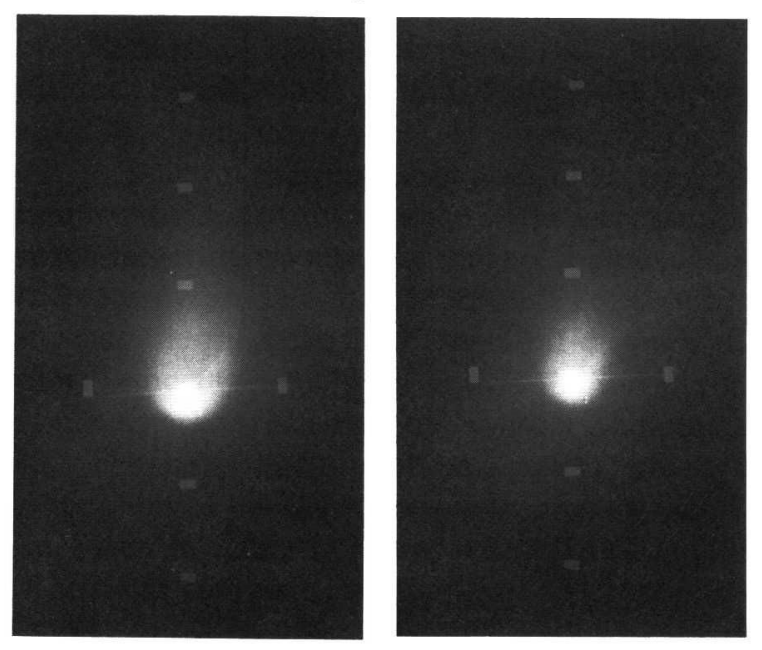

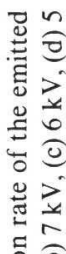

อิ อ

$>\vec{i}$

D

范苛

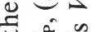

工

뇨

递音

हㅡㄹ

芩品

\&

青

可

今

(3)

e

品
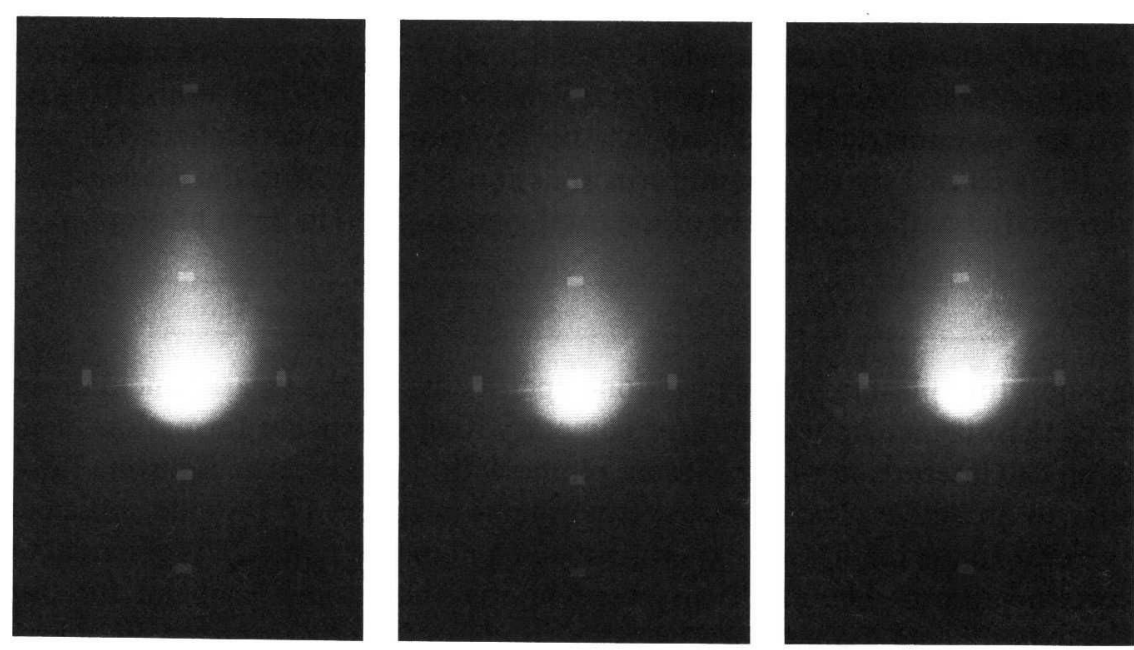

无

¿ 00

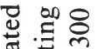

픈 11

范

क

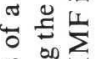

․․․

웛

政

잉ㄱ

욤

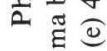

过㧝

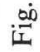




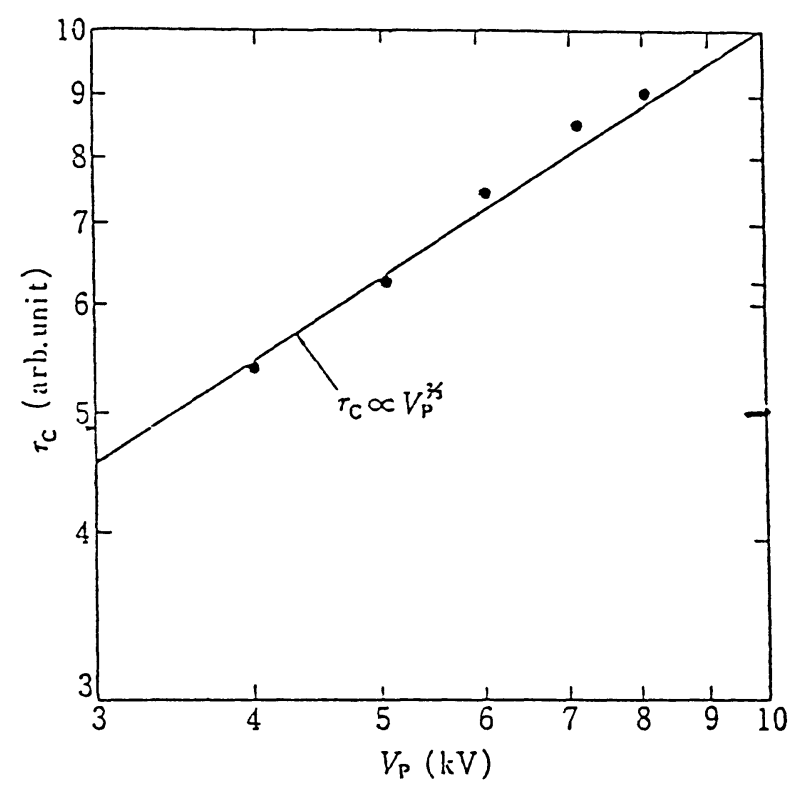

Fig. 15. Experimental stand-off distance versus operating voltage, $V_{\mathrm{P}}$, compared to the slope of $2 / 3$.

In Figs. 11(b) and 12(b), when the plasma plume is injected through a hole perpendicular both to the simulated solar wind plasma flow and the line of sight, the trajectories of the released plasma from the nucleus are shown by illumination which would be applicable for the dynamical observations of such structures called rays (WuRM and MAMMANO, 1967; BRANDT and MENDIS, 1979). These results, also related to an asymmetrical structure of the cometary magnetosphere (IP and MENDIS, 1977) caused by the nonuniform emission of gases from the nucleus, are not easy to predict without the help of the total view as given in these photographs.

\section{Discussion}

Our laboratory plasma plume injection experiments for the measurement of the structure of the magnetosphere and for cometary magnetosphere formation focus attention on the interaction of the plasma plume with the magnetized plasma flow. It is similar to an active plasma release experiment in space. To provide sufficient similarity between the laboratory experiment and a planet or comet, it is essential to satisfy certain criteria. Let us discuss here briefly the scaling problem for our simulation experiments. Laboratory "terrella" studies of magnetospheric reconnection have been carried out by PODGORNY (1976) and PODGORNY et al. (1980), at the Space Research Institute in Moscow, by BAUM and BRATENAHL (1982a) in the T-1 device, and by MINAMI et al., (1977) and MINAMI and TAKEYA (1985) at Osaka City University. Each experiment is designed to satisfy the principle of limited simulation 
which allows a certain group of events (such as magnetospheric reconnection, energy transfer, etc.), to be modeled within the appropriate plasma regime. BARANOV (1969) derived and discussed the significance of non-dimensional simulation ratios (for a detailed discussion, see BAUM and BRATENAHL, 1982a; MINAMI and TAKEYA, 1985).

Each simulation fulfills the following prescription: the magnetopause stand-offdistance considerably exceeds the model earth size (several $\mathrm{cm}$ ), the Alfven and sonic Mach numbers (2-10) are much larger than unity, the solar wind duration (100 $\mu \mathrm{s}$ ) is many magnetosphere transit times (several $\mu \mathrm{s}$ ) long, the magnetic Reynolds number divided by the product of the ion gyrofrequency and the ion collision time is much larger than unity, the magnetopause stand-off-distance (several $\mathrm{cm}$ ) is larger than one gyroradius, and the ion collisional mean free path (10 to several tens of $\mathrm{cm}$ ) exceeds the magnetopause stand-off-distance. For the simulation experiment the most important condition is that the ion gyroradius should be smaller than the system characteristic size, otherwise it is impossible to get the fine structure.

The non-dimensional parameters which define various criteria governing simulation of the earth's magnetosphere by UCR-T1 and at Osaka City University are given in Table 1. MINAMI and TAKEYA (1985) indicated that the criterion of $R / \lambda_{\mathrm{i}}<1$ is too restrictive because observations were made in the moving plasma frame.

For limited simulation of the artificial (AMPTE) and actual cometary magnetospheres, MINAMI et al. (1986) stressed the importance of the ratio of (stand-off distance, $R) /\left(\right.$ ion mean free path, $\lambda_{\mathrm{i}}$ ). The value $R / \lambda_{\mathrm{i}}=(3 \mathrm{~cm} / 50 \mathrm{~cm})$ should be $\ll 1$. Magnetization of the plasma flow by the IMF, makes the mean free path effectively small (nearly the ion gyroradius, $R_{\mathrm{G}}$ ). So $R_{\mathrm{G}} / R(1 \mathrm{~cm} / 3 \mathrm{~cm}) \ll 1$ is also required to demonstrate the important role of the IMF for cometary magnetosphere formation (Figs. 11 and 12). When the IMF is weaker than $50 \mathrm{G}$, the cometary simulation must

Table 1. The non-dimensional parameters of the experiments.

\begin{tabular}{ccccc}
\hline Parameter & Space & SRIM $^{1}$ & $\mathrm{UCR}^{2}$ & $\mathrm{OCU}^{3}$ \\
\hline$R / r>1$ & 10 & 4.9 & 10 & 2.4 \\
$M_{\mathrm{A}}>1$ & 9.4 & 7.7 & 7.7 & 2 \\
$M_{\mathrm{i}}>1$ & 20 & 6.9 & 6.9 & 10.6 \\
$1 / A_{0 \mathrm{i}}=V \Delta t / R \gg 1$ & $\infty$ & 10.2 & $1 \times 10^{2}$ & 10.5 \\
$A_{1 \mathrm{i}} M_{\mathrm{i}}=R / L_{\mathrm{i}} \gg 1$ & $1.9 \times 10^{3}$ & 1.9 & 1.3 & 1.2 \\
$A_{2 \mathrm{i}} M_{\mathrm{i}}=R_{\mathrm{m}} / \omega_{\mathrm{i}} \tau_{\mathrm{i}} \gg 1$ & $8.0 \times 10^{5}$ & $5 \times 10^{3}$ & $3.5 \times 10^{3}$ & $4.7 \times 10^{4}$ \\
$A_{3 \mathrm{i}}=R / \lambda_{\mathrm{i}} \ll 1$ & $4 \times 10^{-5}$ & 0.4 & 0.27 & 8.5 \\
\hline
\end{tabular}

Where $R$ is the distance to the sub-solar magnetopause; $M_{\mathrm{A}}$ and $M_{\mathrm{i}}$ the Alfven and ion thermal Mach numbers based on $\left(k T_{\mathrm{i}} / m_{\mathrm{i}}\right)^{1 / 2} ; L_{\mathrm{i}}$, the ion gyroradius; $R_{\mathrm{m}}$ is the magnetic Reynolds number based on $R$ and the Spitzer Conductivity $\left(R_{\mathrm{m}}=2.4 \times 10^{3}\right.$ for UCR, and $4 \times 10^{2}$ for OCU); $\omega_{\mathrm{i}}, \tau_{\mathrm{i}}$, the ion gyrofrequency and ion collision time; $\lambda_{\mathrm{i}}$, the ion collision mean free path.

${ }^{1}$ After Podgorny (1976) and Podgorny et al. (1980), ${ }^{2}$ after Baum and Bratenahl (1982a), and ${ }^{3}$ after MinAMI and TAKEYA (1985). 
be limited. For the cometary simulation, the size ratio $R_{X} / R$ where $R_{X}$ is the obstacle size is also important. If $R_{X} / R$ is near unity, the shock formation by the direct plasma-solid body interaction dominates the comet-type plasma-plasma interaction. A part of the confusion between comet and Venus-type interactions could be avoided by checking the value of $R_{X} / R$.

One characteristic of such a plasma injected into the magnetized plasma is a diamagnetic effect. HAERENDEL (1973) observed the diamagnetic effect of the earth's magnetic field during the formation of an artificial plasma by a chemical release experiment aboard a sounding rocket (Fig. 16). They found that the diamagnetic effect was broken earlier than predicted by the classical magnetic field diffusion into the plasma. This limited diffusion/confinement time relates not only to the magnetic reconnection in the earth's magnetosphere and the merging of luminous cometary plasma tails in space (MINAMI, 1987b), but also to the confinement of fusion plasmas. BAUM and BRATENAHL (1982b, 1983b) discussed the importance of such diamagnetic effect to check the "frozen-in condition" of the solar wind plasma near the earth's orbit and obtained an experimental result in the UCR-T1 device for the interaction of a plasma stream with a transverse magnetic

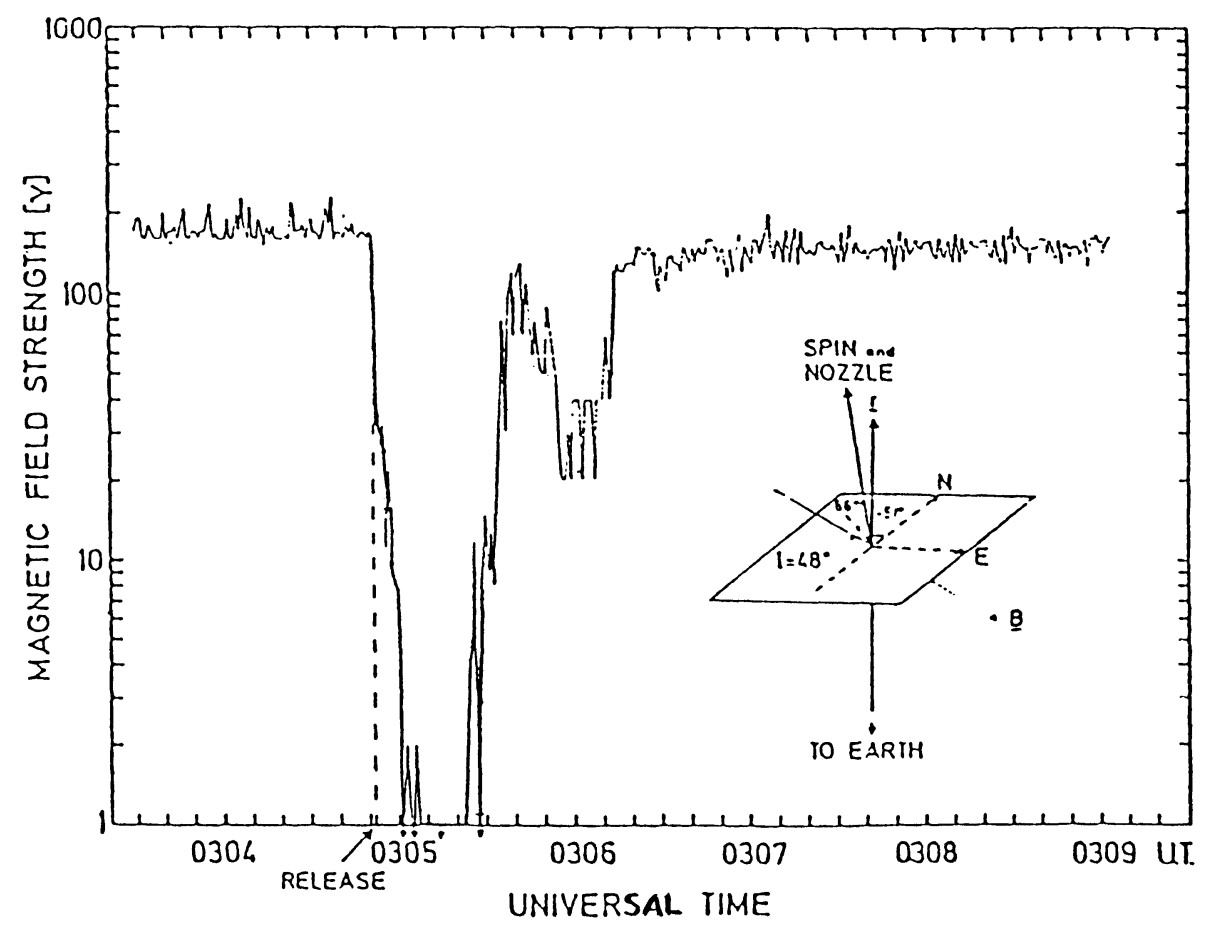

Fig. 16. Magnetic field strength as measured with a search coil magnetometer on a Scout rocket during release of the barium cloud. The measured field is corrected for the known angle between magnetic field and spin axis of the rocket (after HAERENDEL (1973)). 


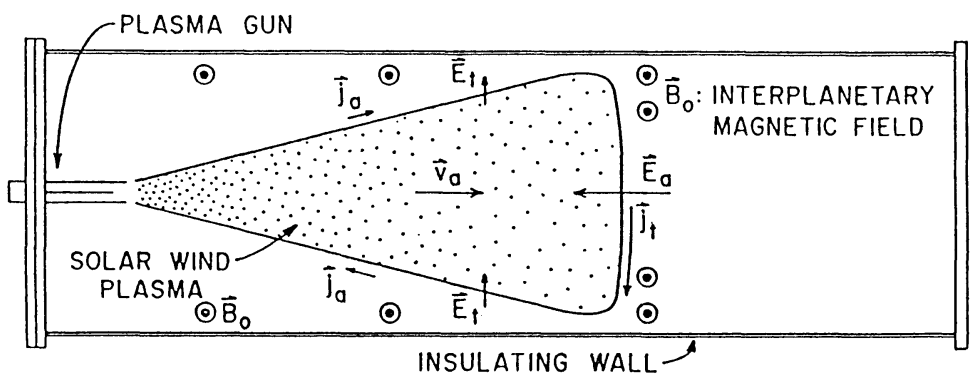

Fig. 17. A coaxial plasma accelerator projects a fast plasma from left to right across a transverse magnetic field of external origin which points into the page. Transverse and axial (relative to the stream velocity) electric fields and "shielding" currents for diamagnetization are estimated. The stream expands as it leaves the accelerator and decreases in density.
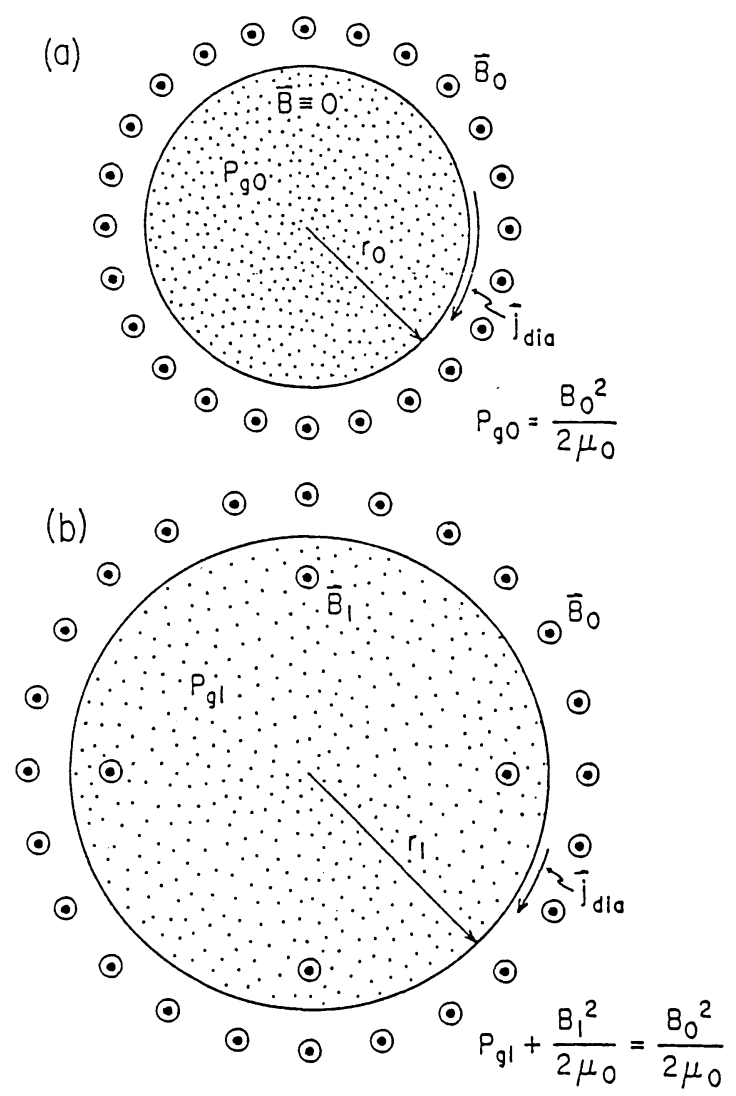

Fig. 18. (a) Static equilibrium where an unmagnetized plasma of gas pressure $P_{g 0}$ is balanced by magnetic pressure of $B_{0}$. (b) A similar static equilibrium in which the plasma is weakly magnetized with $B_{1}$. According to MHD both states can exist but a transition between (a) and (b) could not occur if the frozen-in theorem were valid. For the solar wind $B_{\mathrm{g} 1} \gg \boldsymbol{B}_{0}$, so a comparative study of the magnetized flow with the actual solar wind would give us the origin of the magnetization of the solar wind and a check on the frozen-in condition. 


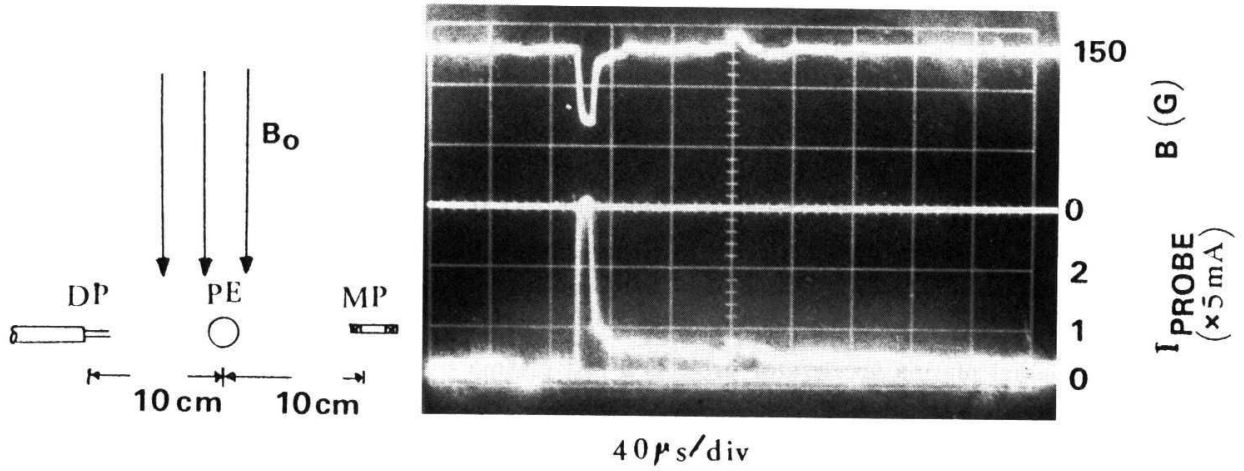

Fig. 19. An example of the measured diamagnetic effect of the injected plasma plume surrounded by the uniform magnetic field $B_{0}(150 \mathrm{G})$ (upper trace). The plasma density inside the boundary measured by a double probe is also shown at the bottom trace. The location of the double probe (DP) and magnetic probe (MP) each separated by $10 \mathrm{~cm}$ from the plasma emitter (PE) is shown.

field (Figs. 17 and 18). The results indicate that the magnetic field $B_{g 1}$, inside the plasma flow with externally applied magnetic field, $B_{0}$, as found from the pressure balance equation, may have any degree of diamagnetism between the extreme limits of perfect and zero diamagnetism.

The time required for stream magnetic confinement agrees approximately with an estimate based on diffusion due to Hall conductivity or gyroconductivity. Figure 19 shows an example of the measured diamagnetic field inside the injected plasma plume used for comet simulation with an externally applied magnetic field of $150 \mathrm{G}$. In this case, since the electrical power was applied during the plasma injection, the diamagnetic effect appears to be due to fast recombination in the plasma plume. In the simulation of artificial plasma plume injections and of comets, the magnetic diffusion time is also important.

The structure of magnetic field lines around cometary magnetospheres is measured using conventional two-dimensional $\left(B_{X}, B_{Z}\right)$ magnetic probes. The results show an enhancement of $B_{X}$ near the cometary boundary and a decrease in $B_{Z}$. When used in conjunction with magnetic probes, the mapping of magnetic field lines by plasma injections becomes a powerful method of understanding the magnetospheric structure.

One of the authors (S. Minami) wishes to express his thanks to N. Kawashima of the ISAS (Institute of Space and Astronomical Sciences) for his valuable discussions and A. Bratenahl for his warmest encouragement during the author's stay at the Institute of Geophysics and Planetary Physics, University of California, Riverside.

This work was supported under Grants NASA ATM-80-18315, NAGW-709, ATM-8214046, CSI \#cs-33-81. 


\section{REFERENCES}

BARANOV, V. B., Simulation of flow of interplanetary plasma past the magnetosphere of the earth or planets, Cosmic Res., 7, 98-104, 1969.

BAUM, P. J., Laboratory simulation of magnetic reconnection; Chapter 1, NASA, Solar Terrestrial Phys.-Present and Future, Proc. Solar-Terrestrial Phys. Workshop, Berkley Springs, W.VA, Chap. 1, pp. 26-31, NASA, 1984.

Baum, P. J. and A. Bratenahl, The laboratory magnetosphere, Geophys. Res. Lett., 9, 435-438, 1982 a.

BAUM, P. J. and A. BRATENAHL, Rapid magnetic flux transport in laboratory and space plasmas, Report UCR/IGPP-82-7, 24 pp., 1982b.

BAum, P. J. and A. Bratenahl, Reconnection in terrella experiments, Chapman Conference on magnetic reconnection, LANL, 19 pp., 1983a.

Baum, P. J. and A. Bratenahl, Interaction of a plasma stream with a transverse magnetic field: Experimental study of the plasma magnetization in the absence of short circuits and the Bohr wall effect, Report UCR/IGPP-83-22, 15 pp., 1983b.

Birkeland, K., The Norwegian Aurora Polaris Expedition 1902-1903 Volume 1, On the Cause of Magnetic Storms and the Origin of Terrestrial Magnetism, Christina, A. W. Broggers Printing Office, 1908.

Bostick, W. H., M. Brettschneider, and H. Byfield, Plasma flow around a three-dimensional dipole, J. Geophys. Res., 68, 5315-5317, 1963.

Brandt, J. C. and D. A. Mendis, The interaction of the solar wind with comets, in Solar System Plasma Phys. II, pp. 253-292, North Holland Pub. Co., 1979.

Cheng, D. Y., Application of a deflagration plasma gun as a space propulsion thruster, AIAA J., 9, $1681-1685,1971$.

Cladis, J. B., T. D. Miller, and J. R. BAskett, Interaction of supersonic plasma stream with a dipole magnetic field, J. Geophys. Res., 69, 2257-2272, 1964.

Dubinin, M. E. M., I. M. Podgorny, and Yu. N. Potanin, Experimental evidence of opened and closed magnetospheres existence, Rep. Pr-335, 24 pp., Space Res. Inst., Moscow, USSR, 1977.

Dubinin, M. E. M., I. M. Podgorny, and Yu. N. Potanin, On magnetic field structure on the magnetosphere boundary, Rep. Pr-440, 28 pp., Space Res. Inst., Moscow, USSR, 1978.

DUngeY, J. W., The structure of the exosphere or adventures in velocity space, in Geophys, edited by C. Dewitt, J. Hieblot and A. Lebeau, pp. 505-550, Gordon and Breach, Sci. Pub., New York, 1962.

Fukushima, N. and N. Kawashima, Model experiment and neutral phenomenon of interaction of solar plasma stream with geomagnetic field, Rep. Ionos. Space Res., Japan, 18, 4, 1964.

HAERENDEl, G., Results from barium cloud release in the upper atmosphere, Space Res., 1-13-41, 18 pp., 1973.

IP, W.-H. and W. I. AXFORD, Theories of physical processes in the cometary comae and ion tails, Comets, edited by Laurel Wirkening, pp. 588-634, Univ. Arizona Press, 1982.

IP, W.-H. and D. A. MENDIS, The structure of cometary ionosphere, 2. CO rich comets, Icarus, 30, 377-384, 1977.

Kawashima, N., The interaction of a plasma stream with a three-dimensional magnetic dipole, J. Phys. Soc. Japan, 18, 227-234, 1964.

Kawashima, N. and S. MORI, Laboratory experiments on the intrusion of plasma in a simulated magnetic cavity II,--path of intruding plasma, JAERI Memo 2513, Jpn. Atom. Energy Res. Inst., 1966.

Minami, S., Digitized photographs of a simulative magnetosphere, EOS, 68, 699, 1987a.

Minami, S., Large scale structure of cometary plasma tails, Proc. 20th ISAS Lunar and Planetary Symposium, pp. 47-50, ISAS, Tokyo, 1987b.

MiNAMI, S. and S.-I. AKASOFU, Laboratory simulation of the interplanetary magnetic field effects on the magnetosphere, Planet. and Space Sci., 34, 987-992, 1986.

MinAmi, S. and S.-I. AKasofu, Laboratory evaluation of energetic coupling between the solar wind and the magnetosphere, in Laboratory and Space Plasmas, edited by H. Kikuchi, Springer Verlag., 1988 
(in press).

MINAMI, S. and Y. TAKEYA, Flow of artificial plasma in a simulated magnetosphere: Evidence of direct interplanetary magnetic field control of the magnetosphere, J. Geophys. Res., 90, 9503-9520, 1985.

Minami, S. and Y. TAKeYA, Plasma injection into a simulated magnetosphere, Planet. and Space Sci., 34, 69-76, 1986.

Minami, S. and R. S. White, An acceleration mechanism for cometary plasma tails, Geophys. Res. Lett., 13, 849-852, 1986.

Minami, S., Y. Hirose, and Y. TAKeYA, Simulation experiment of twined plasma produced by powered double probe in the tail region of the magnetosphere, Mem. of Fac. of Eng. Osaka City Univ., 18, 27-36, 1977.

Minami, S., P. J. Baum, G. Kamin, and R. S. White, Laboratory formation of a simulated comet, Geophys. Res. Lett., 13, 884-887, 1986.

Minami, S., P. J. Baum, G. Kamin, and R. S. White, Laboratory comet simulation, in Laboratory and Space Plasmas, edited by H. Kikuchi, Springer Verlag., 1988 (in press).

Osborne, F. J. F., M. P. BAChynski, and J. V. Gore, Laboratory studies of the variation of the magnetosphere with solar wind properties, J. Geophys. Res., 69, 4441-4449, 1964.

Podgorny, I. M., Laboratory experiments (Plasma intrusion into the magnetic field), Rep. Pr-225, Space Res. Inst., 1976.

Podgorny, I. M., E. M. Dubinin, and Yu. N. Potanin, On magnetic curl in front of the magnetosphere boundary, Geophys. Res. Lett., 7, 247-250, 1980.

Tomita, K.-I., T. Saito, and S. Minami, Structure and dynamics of the plasma tail of comet Halley, Part I, Kink event on January 10-11, 1986, Astronom. Astrophys., 187, 215-219, 1987.

Valenzuela, S., G. Haerendel, H. Foeppl, F. Melzner, H. Neuss, E. Rieger, J. Stoecker, O. BArer, H. Hoefner, and J. Loidl, The AMPTE artificial comet experiments, Nature, 320, 700-723, 1986.

Wurm, K. and A. Mammano, Dissoziation und Ionisation in Kometen, Icarus, 6, 281-291, 1967. 